\title{
Biological age estimation using an eHealth system based on wearable sensors
}

\author{
Paola Pierleoni ${ }^{1}$ - Alberto Belli ${ }^{1}$. Roberto Concetti ${ }^{1}$. Lorenzo Palma ${ }^{1}$. Federica Pinti ${ }^{1}$ - Sara Raggiunto ${ }^{1}$ (i) \\ Luisiana Sabbatini ${ }^{1} \cdot$ Simone Valenti ${ }^{1} \cdot$ Andrea Monteriù $^{1}$
}

Received: 27 November 2018 / Accepted: 18 November 2019 / Published online: 27 November 2019

(c) The Author(s) 2019

\begin{abstract}
The rapid worldwide aging makes necessary the development of advanced technologies for the objective identification of diseases and disabilities onset. Accordingly, we need to regard the biological age as an alternative and more reliable indicator of the physiological decline of individuals, respect to the simple chronological age. In this paper, we present an eHealth system for estimate the biological age of elderly people starting from the assessment of the frailty phenotype. The frailty phenotype evaluation is made possible using a standard protocol for data acquisition and a cloud application for processing and storing data. The proposed eHealth system is also equipped with wireless, small and non-invasive wearable sensors for an objective evaluation of the mobility of a subject. The eHealth system is tested on a reference population in order to have an amount of data necessary for defining a model to estimate the biological age. The use of the presented system on a reference population, and the availability of data regarding their mobility, allow the validation of the proposed model for the computation of the biological age via simple and objective frailty phenotype assessment.
\end{abstract}

Keywords Biological age estimation $\cdot$ eHealth systems $\cdot$ Frailty phenotype assessment $\cdot$ Wearable sensors

\section{Introduction}

The population of all countries of the European Union (EU) has significantly changed over the years. The rise of life expectations, the increasing health care cost, the caregiver burden and the importance given by people to independent living, justify the development of new assisted life technologies aiming at a safer and self-contained aging. Moreover, the only way to forecast and delay incoming disabilities is the adoption of preventing measures for these people who have higher risk (Cesari et al. 2013). The EU is focused and sensible to these issues linked to the aging society,

Electronic supplementary material The online version of this article (https://doi.org/10.1007/s12652-019-01593-8) contains supplementary material, which is available to authorized users.

Sara Raggiunto

s.raggiunto@pm.univpm.it

Paola Pierleoni

p.pierleoni@univpm.it

1 Department of Information Engineering (DII), Università Politecnica delle Marche, Via Brecce Bianche 12, 60131 Ancona, Italy sharpening the necessity of fast and accurate screening tests for those age-related diseases, some of which are particularly hard to be spot through today's diagnostic modes. Anyway, it is tricky to define how much someone is getting older compared to her/his contemporaries, because life expectation changes from community to community reflecting a mix of genetics, epigenetics, environmental and random factors. Aging is indeed a complex biological process, characterized by the progressive cell and organs deterioration that in turn causes the decline of biological functions (Weidner and Wagner 2014). The most effective mean to reduce disease burden and to control costs is to delay this progression by extending the health span, the period of human life during which the individual is healthy and free from serious diseases, hence the urgent need for biological measures of age.

Indeed, the human body has two different ages, the chronological age (CA) and the biological age (BA). The former depends on the birth date while the latter represents the age based on the biological quality and functioning of tissues, apparatus and organs of an individual. The CA is not a reliable indicator of the physiological decline, such that people with the same CA could show significant differences in their health status (Levine 2012). For 
this reason we need to take into consideration the BA to accurately forecast the course of diseases and the mortality rate. Therefore, knowing a precise BA index for each person is extremely important to be able to identify aging sources, to estimate rejuvenation therapies and to beforehand intervene. The BA is commonly associated to the "real" human age because it reflects the health status and the psycho-physical deterioration of a body. It is capable of detecting individuals at risk and of forecasting disabilities or even death, reason why this concept is so dear to the scientific community (Karasik et al. 2005).

Over the time several studies have tried to find a method to estimate the BA, but still today we lack a universally trusted and accepted golden standard. Nowadays, main methodologies employed to esteem the BA are based on the measurement of biomarkers that are strictly linked to the CA. The World Health Organization (WHO) defines the term biomarker in a broad sense as a word that "includes almost any measurement reflecting an interaction between a biological system and an environmental agent, which may be chemical, physical or biological". Nevertheless, the complexity of the aging process has made impossible the identification of a unique biomarker able to give an accurate measure of the biological aging rate.

Scientific literature provides several possible methods and algorithms that exploit biomarkers to estimate the BA, such as the multiple linear regression (MLR) and the principal components analysis (PCA). Recent studies added new molecular biomarkers based on the DNA and RNA to the classical phenotypic biomarkers usually adopted in scientific research. Among these new biomarkers, we find as a trustworthy predictor of BA, the DNAmAge (also said epigenetic clock) that measures the age of the DNA's methylation. Nowadays, the most powerful predictors of the BA are two of these molecular measures, namely the Horvath's clock and the Hannum's clock (Jylhävä et al. 2017).

Marioni et al. (2016) has been the first to identify association between blood DNAmAge measurements and health status during the aging. In their study they found the cross association of the Horvath's clock with the worsening of the psycho-physical conditions of a subject (Marioni et al. 2015). Breitling et al. (2016) has detected a direct association of the Horvath's clock with the increase of the psychophysical deficits owned by a person. The correlation between BA and deficits accumulation has fostered the definition of two alternative methods to estimate the BA (Searle et al. 2008). These methodologies take into account the psychophysical conditions of a subject and estimate the BA thanks to the analysis of owned deficits and the associated assessment of the frailty. Frailty is commonly described as a state of higher vulnerability to adverse events (Searle et al. 2008) and, in the geriatric medical practice, it is evaluated through deficits count.
Even though the DNAmAge methodology is extremely accurate, it is invasive and expensive too. A non-invasive approach to estimate the BA in elderly people is the one built upon the frailty evaluation, that is commonly made using very complex procedures due to the necessity of continuous monitoring of the psycho-physical conditions of the subject. Anyway, technological progress can support in dealing with the just mentioned issue. Thanks to the information and communication technologies (ICTs) and to the development of wearable and non-invasive sensors, it is possible to reach the 24-h monitoring of subjects still allowing them to do things of daily life without annoyance. Moreover, in this prospective scenario, the doctor would be able to remotely supervise the patient accessing to the gathered data and checking for any variations through the cloud service (Manas et al. 2018). This kind of application is included in the ambient assisted living (AAL) solutions, a flourishing field of research and development. The AAL is born due to the global aging of the population and to the necessity to help elderly persons in living as much as possible in their favourite environment, increasing their self-sufficiency, monitoring their actions and providing assistance (Calvaresi et al. 2017).

Our research project arises in this context, with the aim of finding a non-invasive methodology for the BA evaluation in elderly people. In particular, we propose a complete eHealth system subject based on commercial devices, wearable sensors, smartphone application, and cloud services, usable in both outpatient and home monitoring. Below we are going to present the characteristics of the developed eHealth system, that supports the frailty assessment procedure thus allowing the BA evaluation.

\section{Biological age and frailty evaluation}

It is not easy to understand directly the BA of a subject, as previously pointed out, reason why largely diffused methodologies to compute a reliable measurement of BA start from the frailty estimation (Karasik et al. 2005). Conceptually, frailty is a diagnosable state, typical of elderly people, characterized by an increased vulnerability, caused by age-related declining that undermines daily capabilities. Specifically, frailty could even be present without any other detected pathological condition (Cesari et al. 2013). Several studies have theoretically addressed this topic, ending up that frailty is a tangible geriatric syndrome, characterized by low reserves and poor resistance to stressors, associated with an higher risk of bad outcomes for the subject's future health (Ensrud et al. 2018).

In literature, the most exploited methodology of BA estimation among the frailty-based approaches, is the one grounding on the frailty index (Mitnitski et al. 2001). The frailty index 
" $q$ " can be quantified after a complete geriatric evaluation of the patient, because it depends on the count of current deficits through multiple domains (cognition, mood, mobility, chronic diseases and so on). In particular, it is computed as the ratio between current deficits of a subject respect to the total number of deficits that have been taken into account during the geriatric evaluation (Searle et al. 2008). The study carried out by Rockwood and Mitnitski (2007) estimates the biological age of a group of Canadian over-65 people, exploiting the Frailty Index computed considering a total amount of 20 deficits. Starting from the average values of the frailty index and using the linear regression, Mitnitski obtained a mathematical model and extracted from it the log-linear behaviour of the frailty index in relation to the CA of the subject (Mitnitski et al. 2002). The mathematical model described by Mitnitski, depicted in Fig. 1, matches the following equation:

$\ln (q)=-4.23+0.03 \cdot C A$.

From the mathematical model, it is possible to observe that the increasing count of deficits that turns into an higher Frailty Index for older subjects (higher $C A$ ) (Fig. 1). Applying an inverse regression to the Mitnitski's model, we can derive the personal biological age (PBA) employing the following equation:

$P B A=126+26.09 \cdot \ln (q)$,

where PBA represents the average value for which certain deficits are present in the reference group of people ageing well.
The frailty index methodology, although precise and accurate for its detailed risk scale, has some drawbacks, such as the large number of deficits to be taken into account (at least 30/40), the lack of an unequivocal definition of them (Bergman et al. 2007; Rockwood and Mitnitski 2007) and the dependency of the estimation on a complete geriatric evaluation, something that can be carried out by professional medical staff only (Guaraldi et al. 2017).

An alternative way to evaluate the frailty of a subject is built upon the Frailty Phenotype concept, that has been introduced by Fried. In this study the frailty was classified based on five phenotypical criteria: weight loss, grip strength, poor endurance and energy, walk time and physical activity (Fried et al. 2001). Thanks to this criteria-based definition of frailty, subjects can be easily and quickly evaluated for what it concerns their physical and psychological condition. The five criteria are defined as predictors of frailty (Fried et al. 2001) and, specifically, we can identify a frail subject (if accomplished three or more criteria), a pre-frail one (if accomplished one or two criteria) or a non-frail one (no criterion accomplished). The proposed model underlines the cyclic nature of frailty and shows how functional losses, in one or more areas, could let the functional decline of the whole body start. Fried's study has uniquely defined every criterion with the associated cut-off values as follows:

1. Weight loss: unintentional weight loss, not due to dieting or exercise, calculated as:

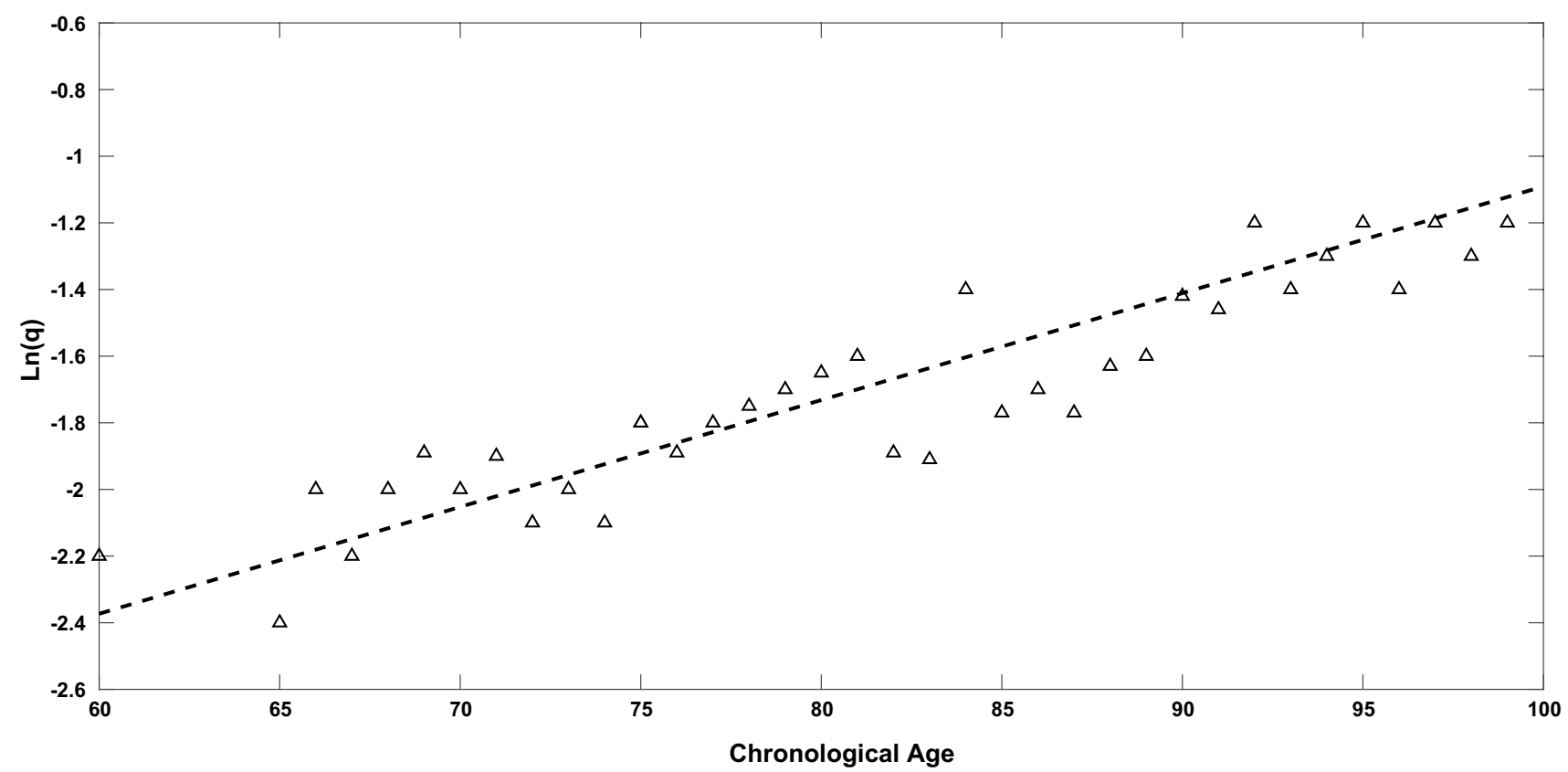

Fig. 1 Biological age estimation using the model based on frailty index 
Table 1 Cut-off values beyond satisfy the grip strength criterion

Table 2 Scores associated to the question: "How often in the last week did you feel this way?" which the subject does not

\begin{tabular}{lll}
\hline Gender & BMI & Cut-off $(\mathrm{kg})$ \\
\hline Man & $\leq 24$ & 29 \\
& $24.1-28$ & 30 \\
& $\geq 28.1$ & 32 \\
Woman & $\leq 23$ & 17 \\
& $23.1-26$ & 17.3 \\
& $26.1-29$ & 18 \\
& $\geq 29.1$ & 21 \\
\hline
\end{tabular}

\begin{tabular}{ll}
\hline Score & How often \\
\hline 0 & $<1$ day \\
1 & $1-2$ days \\
2 & $3-4$ days \\
3 & Most of time \\
\hline
\end{tabular}

$K=\frac{\text { Weight in previous year }- \text { Current measured weight }}{\text { Weight in previous year }}$.

If $K \geq 5$ the individual is considered frail respect to this criterion.

2. Grip strength: maximum grip strength, Table 1 shows the thresholds in kg below which the subject is considered frail. These values depends on the gender of the subject and on the range of her/his body mass index (BMI).

3. Poor endurance and energy: self-report exhaustion level. In order to evaluate this criterion, two specific statements from the Center for Epidemiological StudiesDepression (CES-D) scale (Radloff 1977) are read: "I felt that everything I did was an effort", "I could not get going". Afterwords, it is asked the subject how many times during the last week has she/he ever felt like the just mentioned conditions, and the answer is scored as shown in Table 2. If the score is 2 or 3 then the subject is deemed frail respect to this criterion.

4. Walk time: elderly's mobility monitoring, based on time to walk $4.57 \mathrm{~m}$ at the rhythm chosen on the basis of individual skills. The cut-off parameters are reported in Table 3 as function of the individual's gender and height, above these values the subject is deemed frail.

5. Physical activity: based on self-assessment. Completing the short version of the Minnesota Leisure Time Activity questionnaire, a weighted score of expended kilocalories per week was calculated (considering 18 activities carried out). The cut-off values to discern a frail subject in function of the gender are shown in Table 4.
Table 3 Cut-off values beyond which the subject does not satisfy the walk time criterion

\begin{tabular}{lll}
\hline Gender & Height $(\mathrm{cm})$ & Cut-off $(\mathrm{s})$ \\
\hline Man & $\leq 173$ & 7 \\
& $>173$ & 6 \\
Woman & $\leq 159$ & 7 \\
& $>159$ & 6
\end{tabular}

Table 4 Cut-off values below which the subject is considered frail respect to the physical activity criterion

\begin{tabular}{ll}
\hline Gender & Cut-off $($ kcal $)$ \\
\hline Man & 383 \\
Woman & 270 \\
\hline
\end{tabular}

The frailty phenotype (FP) has been largely investigated and validated by scientific researchers for its capacity of being reproducible in the clinical practice and because it can be evaluated even without the presence of professional medical staff. Moreover, in agreement with what Cesari et al. (2013) has highlighted, the FP may be more suitable than the frailty index for an immediate identification of non-disabled elders at risk of negative events.

Our research aims to propose a non-invasive method for biological age evaluation through the FP assessment of a subject. In our previous study (Pierleoni et al. 2018), we have proposed a methodology to estimate the biological age based on a FP assessment carried out according to Fried's protocol. In this work the data relating to FP assessment were compared with those obtained from the study of Theou et al. (2014) in which the characteristics of frailty have identified, taking into account the frailty phenotype assessment measured of a sample of 27,527 Europeans. However, the study of Theou et al. (2014) only derived the Frailty Phenotype of Europeans without going deeper into the abstraction of a mathematical model useful to estimate the BA of a subject. Otherwise, in our previous work we proposed the application of linear regression techniques to estimate the biological age from data relating to FP assessment based on Fried's protocol. In fact, accessing a certain amount of FP data regarding a reference sample, it is possible to infer a model similar to the one obtained with the frailty index as depicted in Fig. 2. The model obtained from our previous work (Pierleoni et al. 2018) and represented in Fig. 2 can be exploited to identify the BA starting from the evaluation of the FP. The Fig. 2 shows that the point A (outlined by the dashed orange line) can be found starting from the CA and the natural logarithm of the frailty phenotype of the subject. The BA (outlined by the dashed black line) is determined from the abscissa of the point corresponding to $\mathrm{A}$ in the model line. In this study we propose a protocol for the BA evaluation of a subject based on the adoption of an eHealth 


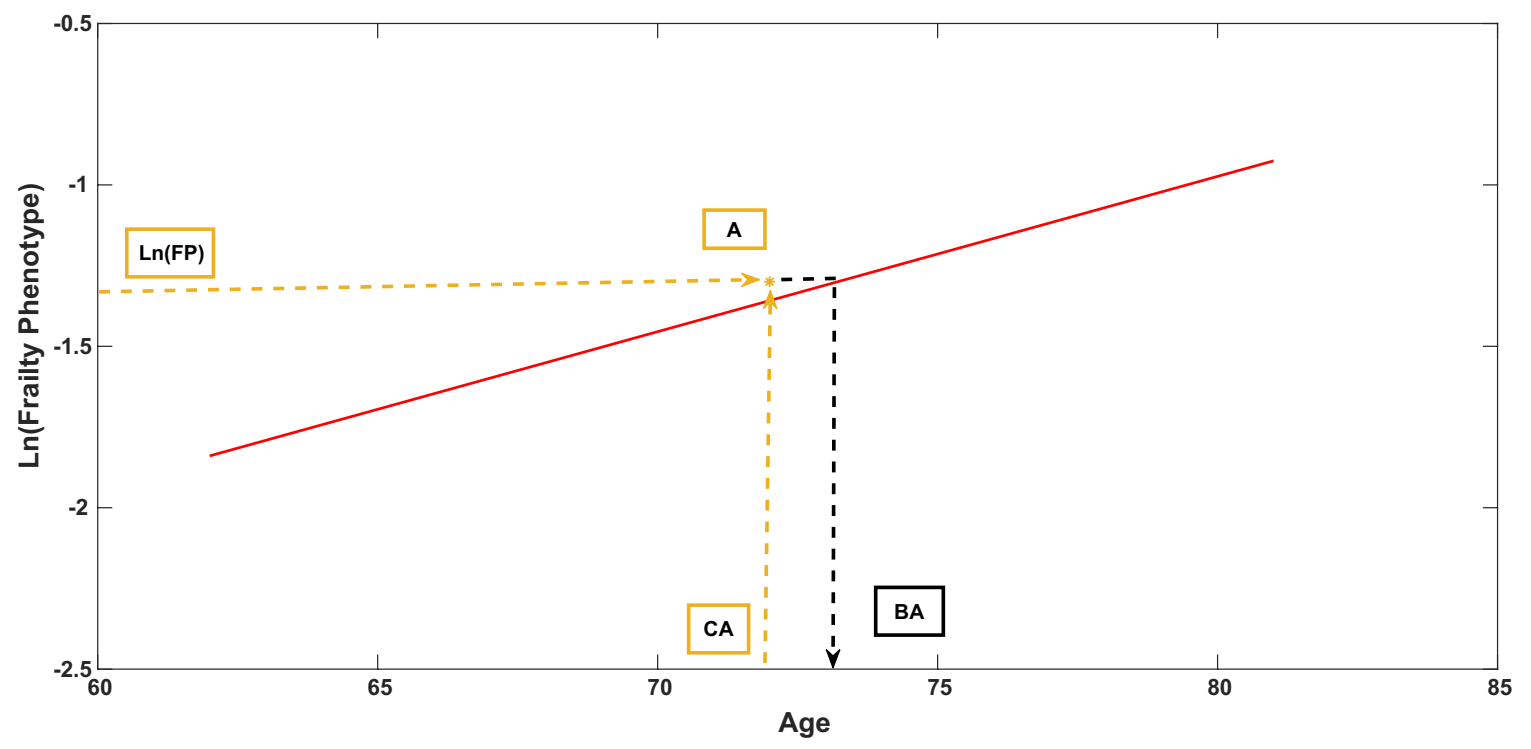

Fig. 2 Biological age estimation using the frailty phenotype assessment

system able to compute the frailty phenotype of the elderly people and provide the biological age of a subject. Respect our previous study, the proposed protocol includes the use of wearable sensors and a smartphone application for the evaluation of the mobility of an elderly subject and the relative phenotypical criterion. Starting from the data acquired in the proposed protocol, the developed eHealth system is able to provides the FP assessment and the BA estimation of a elderly subject, taking into account the FP measured on a reference sample of different age. The results of this work show the FP assessment and the BA estimation providing a comparison between the results obtained from the proposed eHealth system, the outcome of our previous work (Pierleoni et al. 2018) and those derived analyzing data coming from the study of Theou et al. (2014).

\section{System architecture}

A new method to the BA evaluation made through the frailty phenotype analysis is proposed in this work. It has the main advantage respect to the frailty index of taking into consideration only five simple criteria, that still provide a good groundwork for a standardized screening and for the frailty evaluation. To this end, we developed an eHealth system to allow the frailty phenotype measurement on a reference aged population and the subsequent extraction of a mathematical model able to estimate the biological age. Having frailty phenotype data available, regarding a sample population, and exploiting linear regression techniques, it would be possible to extract a robust model able to estimate the BA of each subject starting from the frailty phenotype assessment, as just described in the previous section.

The eHealth system has been created with the aim of making the frailty phenotype evaluation as much objective as possible, and of supplying a user-friendly and automatic solution for the BA estimation to the customers. The method of administration, the results significance and the adoption of efficient supporting tools are essential components to create a reliable method of the $\mathrm{BA}$ estimation that starts from the frailty phenotype evaluation. For this reason, a complete eHealth system has been developed, that is able to take in from different sources the information useful for the frailty phenotype evaluation, to efficiently store data and to allow information analysis thanks to the online upload. Indeed, exploiting cloud computing techniques, the system will enable the medical staff to remotely monitor patients, define personalized treatments and diagnose diseases before they appear.

Figure 3 depicts the eHealth system architecture. The solution has been developed in a way that enables the data storage required for the frailty phenotype analysis, even without direct contact between the subject and the assessor that is evaluating. Specifically, the system allows the upload of data coming from three different sources:

1. Generic user: through a simple user-friendly interface, a registered subject can autonomously register her/his self-evaluation of the frailty phenotype;

2. Medical staff: authorized physicians that can upload evaluation of the frailty phenotype performed on patients, type in additional data, medical report and the 


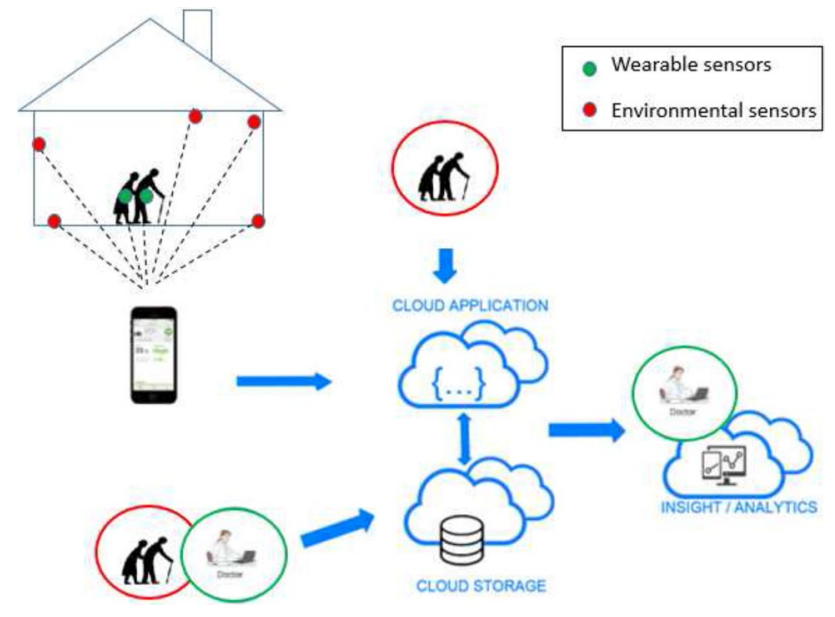

Fig. 3 The eHealth system for data storage and processing

diagnostic-therapeutic path for having a complete medical history of the patient;

3. Distributed sensors: the system allows to upload data coming from specific sensors for the automatic and non-invasive monitoring of the elderly life-style. The integration in the system of wearable and/or environmental sensors is possible only for specific devices, able to access the Internet (Palma et al. 2016) and supplied of entry credentials to a dedicated smartphone that has the objective to aggregate information collected from its associated wireless sensor network.

The eHealth system is made of two parts concerning our objective, that are the cloud application that computes the FP from the results associated to the five frailty criteria and that computes the BA, and the cloud storage that collects the history of each subject and allows new evaluations whenever desired.

The choice of developing a cloud application comes from the necessity of having a system able to scale and to be reliable, flexible and apt to interoperability. We used MEAN, a fullstack JavaScript framework for Web applications whose components are MongoDB, Express, AngularJS and NodeJS. The application developed in this work is exploited to store the history of medical reports and the history of data acquired through the evaluation protocol. Managing these data, it is able to automatically compute results for each criterion (weight loss, poor endurance and energy, grip strength, mobility and physical activity) and to determine the frailty state. This architecture has to manage and store a huge amount of clinical data represented in the Health Level 7 (HL7) format, that is the worldwide accepted standard for the clinical data sharing (Orgun and Vu 2006). For this reason, we use MongoDB, a NoSQL open source database that provides support for systems of JSON with document-oriented style. In order to store and analyze HL7 messages in MongoDB, we need a conversion from XML to JSON format, while for the elaboration of data coming from sensors a conversion from CSV to JSON is required.

The system architecture could foster the evolution in other connected areas, e.g. tele-medicine and tele-rehabilitation. Moreover, together with the wearable sensors included in this work whose efficiency has been already tested, it is possible to add to the system sensors such as the environmental ones, for a constant monitoring and for an higher control on the subject at home.

\section{Proposed protocol}

This work proposes an evaluation protocol to determine the Frailty Phenotype of a reference sample population. Hereafter, for the Frailty Phenotype estimation, we are going to exploit both the methodologies originally proposed by Fried and the wearable devices specifically developed for the gait analysis of a subject. The protocol is made of an initial phase of clinical and personal data gathering for each patient, followed by the next phase where specific tests, named frailty evaluation test (FET), are ran. The FET involves the assessment of the following five criteria to ultimately evaluate the phenotypic frailty risk of elderly people:

1. Weight loss: assessed through an explicit question to the patient or directly measured with a balance.

2. Weakness: measured out thanks to an hand-dynamometer. This test is performed three times by the patient to compute the average value of the measurements.

3. Tiredness: assessed through the CES-D questionnaire administration.

4. Mobility: assessed through the Timed Up and Go (TUG) test. The subject has to stand up from a seat, walk for $5 \mathrm{~m}$, turn around a fixed point and then come back to the seat and sit down (Mathias et al. 1986). This test is performed two times to finally compute the average duration.

5. Physical activity level: assessed through the administration of the Minnesota Leisure-Time Activity questionnaire in its short version. The weekly consumed calories are estimated using the following equation (Taylor et al. 1978):

$\frac{\text { Kcal }}{\text { week }}=$ MET $\cdot \frac{\text { Times }}{2} \cdot \frac{\text { Duration }}{60} \cdot$ Weight,

where metabolic equivalent of task (MET) stands for the unitary energy expenditure associated to a certain physical activity (Haskell et al. 2007), Times represents how many times the activity has been done, Duration is 
the activity lasting in minutes and Weight is the mass of the subject expressed in kilograms.

\section{Wearable sensors for mobility assessment}

The problems related to the mobility of a subject are very common in elderly people due to age-related physiological and musculo-skeletal system changes and to the higher presence of different diseases which negatively affect the postural stability. Within the medical framework, gait and mobility are commonly assessed through simple questionnaires administered by physicians (Tinetti 1986). These kind of investigations do not require sophisticated equipment and are easy to be done, however they have the great disadvantage of being subjective, assessor's experience dependent and of lacking specific gait parameters. The only way to objectively and accurately perform a gait analysis today, is to make the assessment inside specialized labs equipped with expensive and complex measurement systems.

The spreading of wearable devices is linked to countless advantages, as for example their potential exploitation outside of the hospital, the low-cost and the possibility to evaluate more than one subject at once. Moreover, thanks to specific algorithms of data fusion, it could be possible to measure and analyze the gait's kinematics (Aminian 2006). These wearable devices have been exploited in several gait analysis techniques, such as monitoring gait abnormalities and falls-detection thanks to their light weight, smallness, affordability and energy-efficiency (Shin et al. 2007).

The adoption of wearable devices for the acquisition and elaboration of data regarding the mobility is very important for taking care and supporting elderly people (Rockwood and Mitnitski 2007) and therefore also for the evaluation of the frailty phenotype. The use of systems helping the mobility analysis for the frailty phenotype evaluation has been deepened from Schwenk et al. (2015), that for the first time exploited wearable sensors with the final aim of estimate the frailty phenotype. Even though they made an accurate analysis of frailty exploiting wearable sensors, their study do not provide any estimation of the aging process from the results regarding the frailty phenotype. For this reason our aim is to exploit wearable sensors for the FP evaluation and, finally, correlate these results with the biological age. This study is not limited to determining FP but also does intend to provide an estimation of the BA of a subject. In fact, the BA estimation allows to assess the real health state of a subject as it represents the most important indicator of physiological decline.

Based on our previous works (Pierleoni et al. 2014b, 2015), we developed a wireless, small and non-invasive system, to help in the mobility assessment of the frailty phenotype. It is mainly composed of a smartphone and wearable sensors capable of carrying out a mobility analysis of a subject based on Schwenk's indications. Placing the wearable sensors on subject's instep through an elastic, the developed system is able to extract useful metrics of the walk, such as stride length, stride speed, percentage of double support and hip angle. The device is made by triaxial gyroscope, accelerometer and magnetometer, a microprocessor and a unit for the storage and transmission of information. Figure 4 depicts the hardware and the block diagram of the developed system. The microprocessor gathers and processes data coming from the sensors, that can be saved in an SD card and conveyed through the Bluetooth interface to a smartphone. A data fusion algorithm, able to precisely estimate the acceleration and the orientation of the body segment on which they are placed, has been implemented in the microprocessor (Pierleoni et al. 2014a) together with a sensing-unit calibration algorithm.

In order to elaborate the data acquired by the wearable sensors, a specific smartphone application has been developed. Handling acceleration and orientation data collected by the wearable sensor, the smartphone application automatically runs the algorithm implemented for the walk analysis. In particular, through acquired data from the wearable sensors, the developed application estimates three main parameters useful to the mobility assessment: double support, stride speed and stride length (Schwenk et al. 2015). The walked length is obtained through the double trapezoidal integration of the acceleration signal along the direction of the walk. To properly compute the values of speed and

Fig. 4 Hardware and block diagram of the developed system
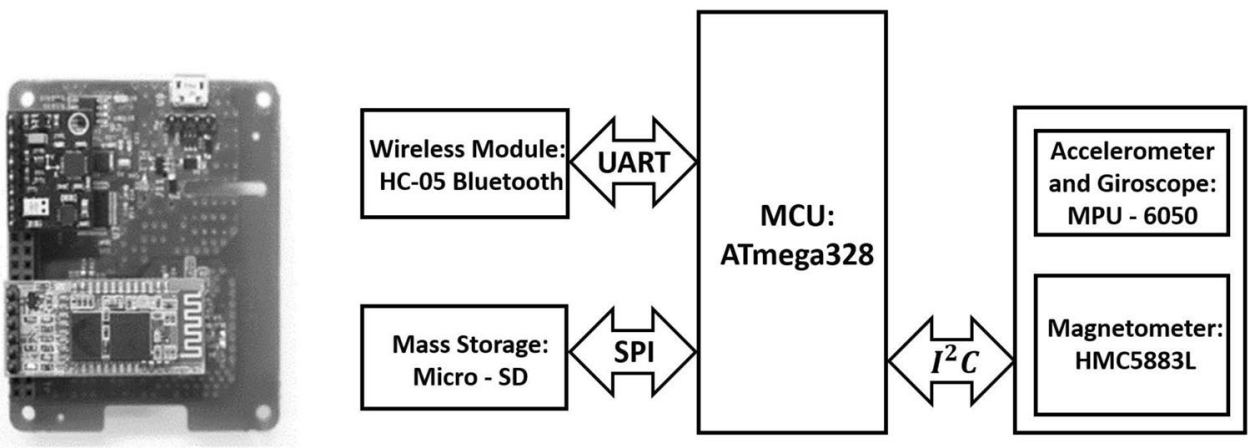
distance, two subsequent filtering phases and two integration phases are necessary. Specifically, the acceleration signal is filtered through a Butterworth high-pass filter of the second order. Speed is then computed as the cumulative integral of the acceleration signal adopting the trapezoidal method, and distance applying a second high-pass filter followed again by the trapezoidal integration. The root mean square (RMS) of the acceleration vector is computed and then its peaks can be determined too. The stride detection is allowed by the fact that each peak corresponds to a stride. Once determined the number of stride, the phases of stance and of swing are evaluated thanks to the determination of local minimums. The relevant points are the initial contact, that is where the stance phase begins and corresponds to the minimum before the highest peak found, and the toe-off, beginning of the swing phase that corresponds to the minimum after the highest peak. The stride length is computed as the distance between two subsequent initial contact belonging to the same foot, once identified the stance and swing phases. Stride speed is then computed as the average for each stride. Finally, the percentage of double support is computed taking into consideration the portion of the stance phase while the two feet are on the ground.
Using the developed smartphone application, the aforementioned parameters are uploaded into the cloud application that processes the data and computes the frailty respect to the mobility criterion. Table 5 shows the thresholds used by the cloud platform to evaluate the frailty related to the mobility criterion. The thresholds of the three parameters taken into account, have been experimentally deducted, as function of the height and gender of the subject. If values stay within the normal range, then the subject is non-frail, otherwise she/he can be frail or pre-frail in case of intermediate positioning of her/his data.

\section{Results}

The main purpose of this work was the development of an efficient methodology for the biological age evaluation starting from a frailty phenotype estimation. The evaluation protocol proposed by us has been applied to 15 elderly ( 8 male and 7 female) aged between 61 and 81 year-old. The results of the performed tests are shown in Table 6 where the gathered data for the frailty phenotype evaluation are illustrated. Table 6 shows the outcome for each of the five criteria as
Table 5 Cut-off values for double support (DS), stride length (SL) and stride speed (SS)

Table 6 Frailty phenotype evaluation results for the reference group of 15 subjects

\begin{tabular}{|c|c|c|c|c|c|}
\hline Parameters & Gender & Height & Non-frail & Pre-frail & Frail \\
\hline Double support DS (\%) & & & $\mathrm{DS} \leq 25$ & $25<\mathrm{DS}<30$ & $\mathrm{DS} \geq 30$ \\
\hline \multirow[t]{2}{*}{ Stride length SL (m) } & & $\leq 165 \mathrm{~cm}$ & $\mathrm{SL} \geq 1.10$ & $0.90<\mathrm{SL}<1.10$ & $\mathrm{SL} \leq 0.90$ \\
\hline & & $>165 \mathrm{~cm}$ & $\mathrm{SL} \geq 1.20$ & $0.95<\mathrm{SL}<1.20$ & $\mathrm{SL} \leq 0.95$ \\
\hline \multirow[t]{4}{*}{ Stride speed SS (m/s) } & Man & $<173 \mathrm{~cm}$ & $\mathrm{SS}>1.0$ & $0.65<\mathrm{SS}<1.00$ & $\mathrm{SS} \leq 0.65$ \\
\hline & & $\geq 173 \mathrm{~cm}$ & $\mathrm{SS} \geq 1.10$ & $0.75<\mathrm{SS}<1.10$ & $\mathrm{SP} \leq 0.75$ \\
\hline & Woman & $<159 \mathrm{~cm}$ & $\mathrm{SS}>1.0$ & $0.65<\mathrm{SS}<1.00$ & $\mathrm{SP} \leq 0.65$ \\
\hline & & $\geq 159 \mathrm{~cm}$ & $\mathrm{SS} \geq 1.10$ & $0.75<\mathrm{SS}<1.10$ & $\mathrm{SS} \leq 0.75$ \\
\hline
\end{tabular}

\begin{tabular}{lllllll}
\hline Patient & Weakness & Weight loss & Tiredness & kcall/week & Mobility & Frailty \\
\hline 1 & - & - & - & - & - & Nonfrail(0) \\
2 & - & - & - & - & - & Nonfrail(0) \\
3 & - & - & - & - & - & Nonfrail(0) \\
4 & - & - & - & Yes & - & Prefrail(1) \\
5 & - & - & - & Yes & - & Prefrail(1) \\
6 & - & - & Yes & - & - & Prefrail(1) \\
7 & Yes & - & - & - & - & Prefrail(1) \\
8 & - & - & - & Yes & - & Prefrail(1) \\
9 & - & Yes & Yes & - & - & Prefrail(2) \\
10 & -- & Yes & - & Yes & - & Prefrail(2) \\
11 & Yes & - & Yes & - & - & Prefrail(2) \\
12 & - & - & - & Yes & Yes & Prefrail(2) \\
13 & - & - & - & Yes & Yes & Prefrail(2) \\
14 & Yes & - & Yes & - & Yes & Frail(3) \\
15 & Yes & - & - & Yes & Yes & Frail(3) \\
\hline
\end{tabular}


defined by Fried and the comprehensive conclusion about the frailty state with the specification of the number of positive-to-frailty criteria in brackets. The subjects have then been arranged for their frailty state. As it can be seen, there are three non-frail subjects, two frail and the remaining are pre-frail. Patients negative to all of the five criteria lead an active life, they have physical activity, have arms stronger than the cut-off values and perform the TUG test in reasonably short time. On the contrary, the identified frail subjects have a weak grip strength, a weekly calories consumption close to the cut-off values and it takes a long time for them to perform the TUG test. Once finished the data gathering phase, the Frailty Phenotype value is estimated for each subject, computed as the average mean weighted for her/his age.

Table 7 contains the results of the TUG test performed exploiting wearable sensors. In detail, it reports the test execution duration, the percentage of double support, the average stride length and the average stride speed. Focusing in the double support data, it normally corresponds to around the $20 \%$ of the stride. It is evident the increment in the percentage when comparing non-frail patients with prefrail ones, and the even higher increment if confronting with the frail people. This observation lead us to think that frail people have an higher necessity of being in the stable phase of the stride cycle, i.e. when both feet stand on the ground. Moreover, it is evident the considerable increment of the test duration and the associated lowering of speed if we focus on these data. Finally, the decrease in the stride length for those people showing at least two frailty criteria can be easily noticed. If we want to find reasons for this, it may be connected to a reduced muscle strength in their lower limb.

Table 7 Results obtained from the algorithm that evaluates the gait parameters

\begin{tabular}{lllll}
\hline Patient & Frailty & $\begin{array}{l}\text { Double sup- } \\
\text { port (\%) }\end{array}$ & $\begin{array}{l}\text { Stride } \\
\text { length (m) }\end{array}$ & Speed (m/s) \\
\hline 1 & Nonfrail(0) & 20.8 & 1.45 & 1.60 \\
2 & Nonfrail(0) & 22.6 & 1.48 & 1.58 \\
3 & Nonfrail(0) & 23.1 & 1.12 & 1.41 \\
4 & Prefrail(1) & 21.8 & 1.36 & 1.40 \\
5 & Prefrail(1) & 25.0 & 1.10 & 1.33 \\
6 & Prefrail(1) & 24.0 & 0.92 & 1.32 \\
7 & Prefrail(1) & 25.7 & 1.22 & 1.28 \\
8 & Prefrail(1) & 26.2 & 0.94 & 1.17 \\
9 & Prefrail(2) & 27.1 & 1.07 & 1.39 \\
10 & Prefrail(2) & 27.0 & 1.00 & 1.30 \\
11 & Prefrail(2) & 28.6 & 0.82 & 1.05 \\
12 & Prefrail(2) & 34.0 & 0.84 & 0.86 \\
13 & Prefrail(2) & 36.1 & 0.85 & 0.81 \\
14 & Frail(3) & 36.3 & 0.67 & 0.94 \\
15 & Frail(3) & 37.4 & 0.69 & 0.64 \\
\hline
\end{tabular}

The study of Theou et al. has been taken as a reference for the validation of our methodology. Their study is the only one that evaluates the Frailty Phenotype employing a reference population comparable to the one considered by our work. Indeed, we evaluated a sample of Italian elderly people, that is a subset of the European aged population considered by Theou et al. in their research.

Figure 5 depicts the comparison of the obtained results. The blue curve represents the frailty phenotype model emerged from Theou's work, the red curve shows the results elaborated by our cloud application following Fried's definition, while the green curve is related to our results adding gait parameters determined thanks to the wearable sensors. It is possible to notice a discrepancy between our results and that from the reference article. This may be caused by the small number of evaluated subjects in this study, or may be related to the better fit embedded in the model found by us that takes into consideration also specific mobility parameters. This last hypothesis should be proven only acquiring more data.

As previously highlighted in Sect. 2, from the Frailty Phenotype data processing, we can extract a model to estimate the BA. Figure 6 depicts the models obtained from our results together with the reference model.

The model proposed in Fig. 6, that estimates BA through the $\mathrm{CA}$ and the natural logarithm of the frailty phenotype of a subject, corresponds to the following equation:

$B A=88.30+22.08 \cdot \ln (F P)$.

\section{Conclusions}

This paper presents a non-invasive methodology to establish the biological age of elderly people through the frailty phenotype evaluation. The proposed solution is composed of an eHealth system useful to evaluate the five phenotypic criteria, already present in literature, with the additional employment of sensors. In detail, we developed small and non-invasive wireless wearable sensors for the assessment of the mobility criterion of the frailty phenotype. Thanks to the integration of the gait analysis algorithm into the eHealth system, we could compute three main parameters, that are double support, stride length and stride speed. Moreover, the cloud application built is able to compute the frailty phenotype from the results of the frailty tests and finally estimate the BA. The performed evaluations are then stored in the cloud to provide a time trend of the subject's aging.

The set method has been validated analyzing data about 15 elderly subjects. Results show that our solution to estimate the BA is comparable with other solutions already existing in literature. However, an extensive acquisition 


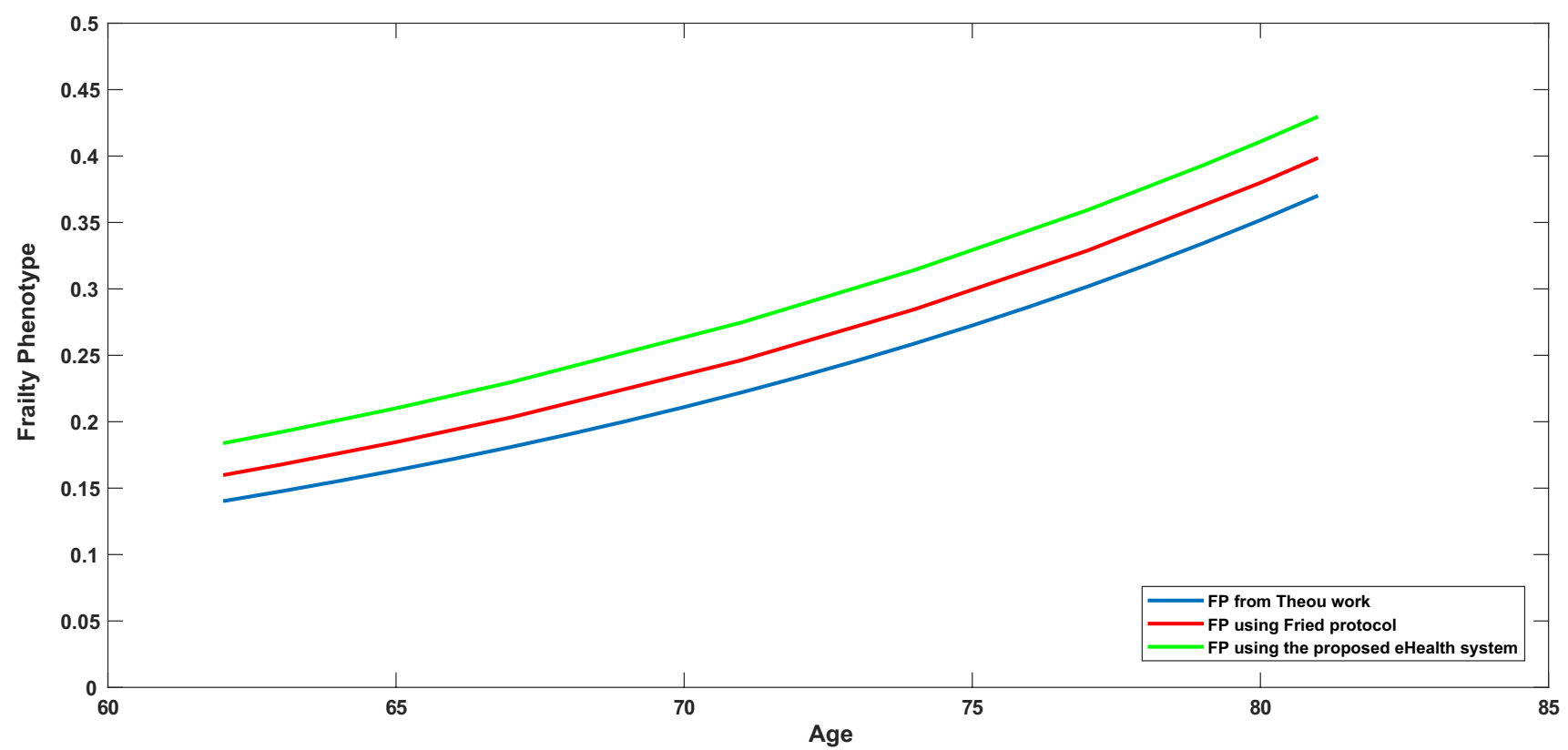

Fig. 5 Comparison of the performance of the frailty phenotype. The blue curve represents the frailty phenotype model emerged from Theou's work, the red curve shows the results elaborated by our cloud

application following Fried's definition, while the green curve is related to our results adding gait parameters determined thanks to the wearable sensors (colour figure online)

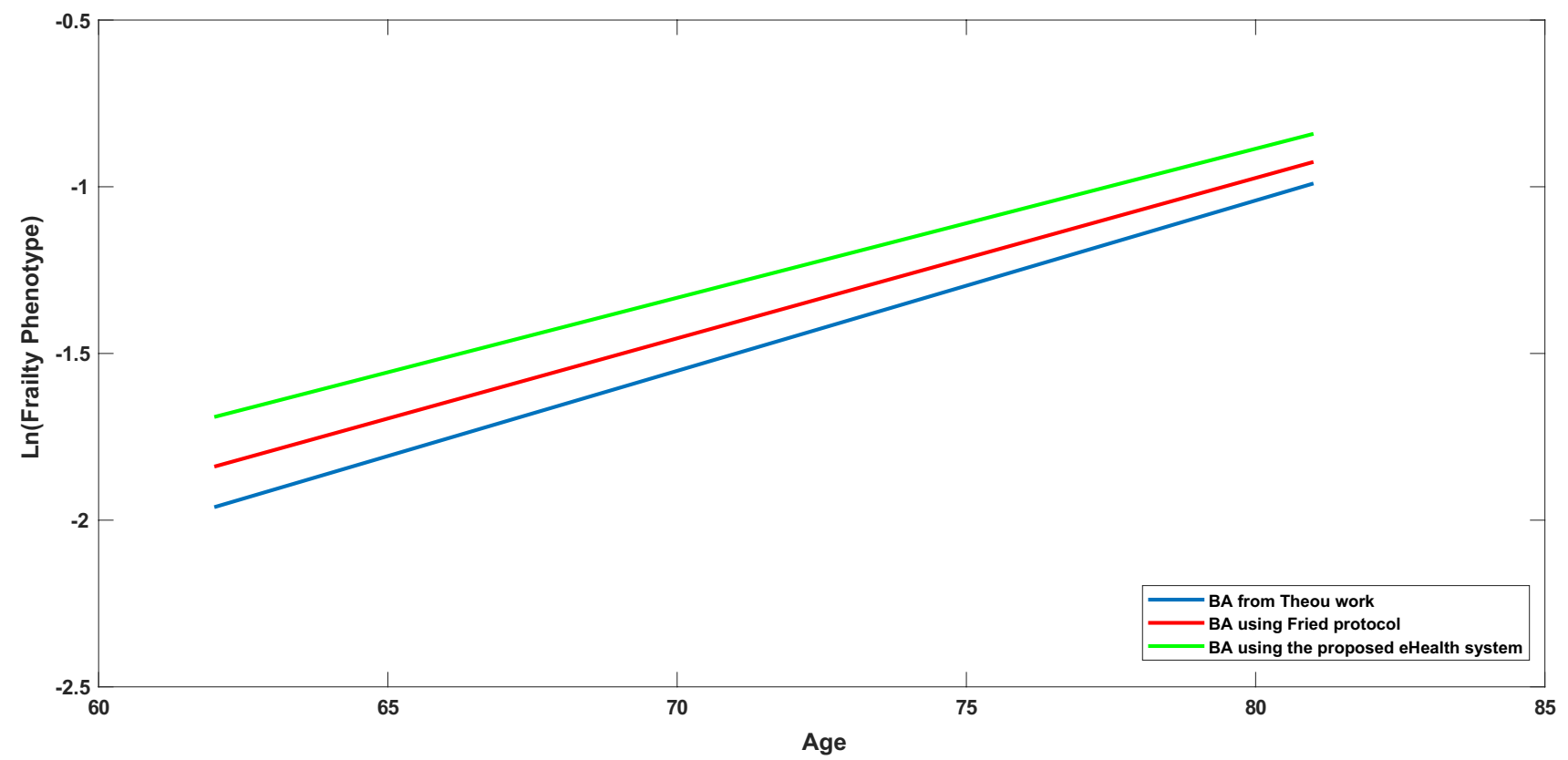

Fig. 6 Comparison of the biological age estimation models. In blue the regression line associated to Theou's study, in red the regression line found following the phenotypic description of frailty and in green the regression line obtained involving wearable sensors (colour figure online)

campaign will facilitate a greater data gathering, thus allowing us to better validate our BA estimation model.
Acknowledgements This study has been promoted within the strategic project entitled "Multidisciplinary Innovative Research Actions on 
Age” (MIR-AGE, CUP: I32F17000080005), funded by the Polytechnic University of Marche, Italy.

Open Access This article is distributed under the terms of the Creative Commons Attribution 4.0 International License (http://creativeco mmons.org/licenses/by/4.0/), which permits unrestricted use, distribution, and reproduction in any medium, provided you give appropriate credit to the original author(s) and the source, provide a link to the Creative Commons license, and indicate if changes were made.

\section{References}

Aminian K (2006) Monitoring human movement with body-fixed sensors and its clinical applications. In: Computational intelligence for movement sciences: neural networks and other emerging techniques, IGI Global, pp 101-138. https://doi.org/10.4018/978-1-59140 -836-9.ch003

Bergman H, Ferrucci L, Guralnik J, Hogan DB, Hummel S, Karunananthan S, Wolfson C (2007) Frailty: an emerging research and clinical paradigm-issues and controversies. J Gerontol Ser A Biol Sci Med Sci 62(7):731-737. https://doi.org/10.1093/gerona/62.7.731

Breitling LP, Saum KU, Perna L, Schöttker B, Holleczek B, Brenner H (2016) Frailty is associated with the epigenetic clock but not with telomere length in a German cohort. Clin Epigenet 8(1):21. https:// doi.org/10.1186/s13148-016-0186-5

Calvaresi D, Cesarini D, Sernani P, Marinoni M, Dragoni AF, Sturm A (2017) Exploring the ambient assisted living domain: a systematic review. J Ambient Intell Hum Comput 8(2):239-257. https://doi. org/10.1007/s12652-016-0374-3

Cesari M, Gambassi G, Abellan van Kan G, Vellas B (2013) The frailty phenotype and the frailty index: different instruments for different purposes. Age Ageing 43(1):10-12. https://doi.org/10.1093/agein g/aft160

Ensrud KE, Kats AM, Schousboe JT, Taylor BC, Cawthon PM, Hillier TA, Yaffe K, Cummings SR, Cauley JA, Langsetmo L et al (2018) Frailty phenotype and healthcare costs and utilization in older women. J Am Geriatr Soc. https://doi.org/10.1111/jgs.15381

Fried LP, Tangen CM, Walston J, Newman AB, Hirsch C, Gottdiener J, Seeman T, Tracy R, Kop WJ, Burke G et al (2001) Frailty in older adults: evidence for a phenotype. J Gerontol Ser A Biol Sci Med Sci 56(3):M146-M157. https://doi.org/10.1093/gerona/56.3.m146

Guaraldi G, Malagoli A, Theou O, Brothers T, Wallace L, Torelli R, Mussini C, Sartini S, Kirkland S, Rockwood K (2017) Correlates of frailty phenotype and frailty index and their associations with clinical outcomes. HIV Med 18(10):764-771. https://doi.org/10.1111/ hiv. 12527

Haskell WL, Lee IM, Pate RR, Powell KE, Blair SN, Franklin BA, Macera CA, Heath GW, Thompson PD, Bauman A (2007) Physical activity and public health: updated recommendation for adults from the American College of Sports Medicine and the American Heart Association. Circulation 116(9):1081. https://doi.org/10.1249/ mss.0b013e3180616b27

Jylhävä J, Pedersen NL, Hägg S (2017) Biological age predictors. EBioMedicine 21:29-36. https://doi.org/10.1016/j.ebiom.2017.03.046

Karasik D, Demissie S, Cupples LA, Kiel DP (2005) Disentangling the genetic determinants of human aging: biological age as an alternative to the use of survival measures. J Gerontol Ser A Biol Sci Med Sci 60(5):574-587. https://doi.org/10.1093/gerona/60.5.574

Levine ME (2012) Modeling the rate of senescence: can estimated biological age predict mortality more accurately than chronological age? J Gerontol Ser A Biol Sci Med Sci 68(6):667-674. https://doi. org/10.1093/gerona/gls233
Manas M, Sinha A, Sharma S, Mahboob MR (2018) A novel approach for IOT based wearable health monitoring and messaging system. J Ambient Intell Hum Comput 1-12. https://doi.org/10.1007/s1265 2-018-1101-z

Marioni RE, Shah S, McRae AF, Ritchie SJ, Muniz-Terrera G, Harris SE, Gibson J, Redmond P, Cox SR, Pattie A et al (2015) The epigenetic clock is correlated with physical and cognitive fitness in the lothian birth cohort 1936. Int J Epidemiol 44(4):1388-1396. https://doi. org/10.1093/ije/dyu277

Marioni RE, Harris SE, Shah S, McRae AF, von Zglinicki T, Martin-Ruiz C, Wray NR, Visscher PM, Deary IJ (2016) The epigenetic clock and telomere length are independently associated with chronological age and mortality. Int J Epidemiol 45(2):424-432. https://doi. org/10.1093/ije/dyw041

Mathias S, Nayak U, Isaacs B (1986) Balance in elderly patients: the" get-up and go" test. Arch Phys Med Rehabil 67(6):387-389

Mitnitski AB, Mogilner AJ, Rockwood K (2001) Accumulation of deficits as a proxy measure of aging. Sci World J 1:323-336. https://doi. org/10.1100/tsw.2001.58

Mitnitski AB, Graham JE, Mogilner AJ, Rockwood K (2002) Frailty, fitness and late-life mortality in relation to chronological and biological age. BMC Geriatr 2(1):1. https://doi.org/10.1186/1471-2318-2-1

Orgun B, Vu J (2006) Hl7 ontology and mobile agents for interoperability in heterogeneous medical information systems. Comput Biol Med 36(7-8):817-836. https://doi.org/10.1016/j.compbiomed .2005 .04 .010

Palma L, Pernini L, Belli A, Valenti S, Maurizi L, Pierleoni P (2016) Ipv6 WSN solution for integration and interoperation between smart home and AAL systems. In: IEEE sensors applications symposium (SAS), pp 1-5. https://doi.org/10.1109/sas.2016.7479840

Pierleoni P, Belli A, Palma L, Pernini L, Valenti S (2014) An accurate device for real-time altitude estimation using data fusion algorithms. In: IEEE/ASME 10th international conference on mechatronic and embedded systems and applications (MESA), IEEE, pp 1-5. https ://doi.org/10.1109/mesa.2014.6935583

Pierleoni P, Belli A, Palma L, Pernini L, Valenti S (2014) A versatile ankle-mounted fall detection device based on attitude heading systems. In: Biomedical circuits and systems conference (BioCAS), 2014, IEEE, pp 153-156. https://doi.org/10.1109/biocas.2014.69816 68

Pierleoni P, Belli A, Maurizi L, Palma L, Pernini L, Valenti S (2015) Performance evaluation of a pedestrian navigation system based on an objective experimental method. In: 12th international workshop on intelligent solutions in embedded systems (WISES), IEEE, pp 41-46

Pierleoni P, Belli A, Concetti R, Palma L, Pinti F, Raggiunto S, Valenti $\mathrm{S}$, Monteriù A (2018) A non-invasive method for biological age estimation using frailty phenotype assessment. In: Italian forum of ambient assisted living. Springer, New York, pp 81-94

Radloff LS (1977) The ces-d scale: A self-report depression scale for research in the general population. Appl Psychol Meas 1(3):385401. https://doi.org/10.1177/014662167700100306

Rockwood K, Mitnitski A (2007) Frailty in relation to the accumulation of deficits. J Gerontolo Ser A Biol Sci Med Sci 62(7):722-727. https ://doi.org/10.1093/gerona/62.7.722

Schwenk M, Mohler J, Wendel C, Fain M, Taylor-Piliae R, Najafi B et al (2015) Wearable sensor-based in-home assessment of gait, balance, and physical activity for discrimination of frailty status: baseline results of the arizona frailty cohort study. Gerontology 61(3):258 267. https://doi.org/10.1159/000369095

Searle SD, Mitnitski A, Gahbauer EA, Gill TM, Rockwood K (2008) A standard procedure for creating a frailty index. BMC Geriatr 8(1):24 https://doi.org/10.1186/1471-2318-8-24

Shin S, Park C, Kim J, Hong H, Lee J (2007) Adaptive step length estimation algorithm using low-cost mems inertial sensors. In: Sensors applications symposium, 2007, SAS'07. IEEE, pp 1-5. https://doi. org/10.1109/sas.2007.374406 
Taylor HL, Jacobs DR Jr, Schucker B, Knudsen J, Leon AS, Debacker G (1978) A questionnaire for the assessment of leisure time physical activities. J Chronic Dis 31(12):741-755. https://doi. org/10.1016/0021-9681(78)90058-9

Theou O, Brothers TD, Peña FG, Mitnitski A, Rockwood K (2014) Identifying common characteristics of frailty across seven scales. J Am Geriatr Soc 62(5):901-906. https://doi.org/10.1111/jgs.12773

Tinetti ME (1986) Performance-oriented assessment of mobility problems in elderly patients. J Am Geriatr Soc 34(2):119-126. https:// doi.org/10.1111/j.1532-5415.1986.tb05480.x
Weidner CI, Wagner W (2014) The epigenetic tracks of aging. Biol Chem 395(11):1307-1314. https://doi.org/10.1515/hsz-2014-0180

Publisher's Note Springer Nature remains neutral with regard to jurisdictional claims in published maps and institutional affiliations. 